\title{
Few-particle eigenstates in the Yukawa model
}

\author{
J.Darewych \\ Department of Physics and Astronomy, York University \\ Toronto, Ontario, M3J 1P3, Canada
}

Received February 13, 1998

\begin{abstract}
We consider a reformulation of the Yukawa model, in which fermions interact via a mediating (massive or massless) scalar field. Covariant Green functions are used express the mediating field in terms of the fermion fields. The resulting Hamiltonian of the theory has an interaction term in which the propagator of the mediating field appears directly. We show that if processes involving emission of physical mediating field quanta can be ignored and an unconventional empty vacuum is used, then exact fewfermion eigenstates of the resulting truncated Hamiltonian can be obtained in the canonical equal-time formalism. These eigenstates lead to two- and three-body Dirac-like equations with scalar interactions. Two-fermion bound states are obtained by the numerical solution of the eigenvalue equation for $J=0$ states. Comparison is made with conventional treatments of this model.
\end{abstract}

Key words: few-fermion systems, Yukawa model, mass spectrum

PACS: 11.10.Qr, 11.10.st

\section{Introduction}

There are few realistic models in the quantum field theory (QFT) for which exact solutions can be written down. In this paper we obtain exact, though unorthodox, few-particle eigenstates in a modified form of the Yukawa model in which fermions interact via a mediating scalar field. The model is of interest because Yukawa couplings are relevant to the quark-Higgs interaction sector of the standard model. The Yukawa model has also been used as a prototype of the FTheoretic description of internucleon interactions, and generalizations that also contain a vector, pseudoscalar, etc. boson exchange, continue to be useful in nuclear physics.

The Yukawa model with a scalar boson exchange is based on the Lagrangian 
density $(\hbar=c=1)$

$$
\mathcal{L}=\sum_{k=1}^{N} \bar{\psi}_{k}(x)\left(\mathrm{i} \gamma^{\mu} \partial_{\mu}-q_{k} \chi(x)-m_{k}\right) \psi_{k}(x)-\frac{1}{2} \partial_{\alpha} \chi(x) \partial^{\alpha} \chi(x)-\frac{1}{2} \mu^{2} \chi^{2}(x),
$$

where $N$ is the number of fermion types. The corresponding classical equations of motion are the $N$ Dirac equations:

$$
\left(\mathrm{i} \gamma^{\mu} \partial_{\mu}-m_{k}\right) \psi_{k}(x)=q_{k} \chi(x) \psi_{k}(x)
$$

in which the boson field serves as a potential, and the Klein-Gordon equation for the mediating boson field,

$$
\partial_{\mu} \partial^{\mu} \chi(x)+\mu^{2} \chi(x)=\rho(x)
$$

in which the source density is provided by the fermion fields:

$$
\rho(x)=\sum_{k=1}^{N} q_{k} \bar{\psi}_{k}(x) \psi_{k}(x)
$$

As it is well known from the electromagnetic theory [1,2], equation (3) has the formal solution

$$
\chi(x)=\chi_{0}(x)+\int D\left(x-x^{\prime}\right) \rho\left(x^{\prime}\right) \mathrm{d}^{4} x^{\prime},
$$

where $D\left(x-x^{\prime}\right)$ is a Green function (or a boson propagator in the QFT terminology), defined by

$$
\partial_{\mu} \partial^{\mu} D\left(x-x^{\prime}\right)=\delta^{4}\left(x-x^{\prime}\right),
$$

and $\chi_{0}(x)$ is a solution of the homogeneous (or "free field") equation (3) with $\rho(x)=0$. Equation (6) does not define the covariant Green function $D\left(x-x^{\prime}\right)$ uniquely. One can always add a solution of the homogeneous equation $\partial_{\mu} \partial^{\mu} D(x-$ $\left.x^{\prime}\right)=0$. A unique $D\left(x-x^{\prime}\right)$ requires specification of the boundary conditions or, in the momentum representation, specification of the contour of the integral. This is discussed in standard texts (e.g. [1,2]). A momentum space representation is

$$
D\left(x-x^{\prime}\right)=\frac{1}{(2 \pi)^{4}} \int \mathrm{d}^{4} k \frac{\mathrm{e}^{-i k \cdot\left(x-x^{\prime}\right)}}{\mu^{2}-k \cdot k+\mathrm{i} \epsilon},
$$

where $D$ is known as the principal value Green function if $\epsilon=0$, and the Feynman propagator if $\epsilon>0$.

Substitution of the formal solution of the mediating-field equation (5) into fermion equations (2) modifies the latter to the set of coupled nonlinear equations:

$$
\left(\mathrm{i} \gamma^{\mu} \partial_{\mu}-m_{k}\right) \psi_{k}(x)=q_{k}\left(\chi_{0}(x)+\int \mathrm{d}^{4} x^{\prime} D\left(x-x^{\prime}\right) \rho\left(x^{\prime}\right)\right) \psi_{k}(x) .
$$


The reduced equations (8) are derivable from the stationary action principle

$$
\delta S\left[\psi_{k}\right]=\delta \int \mathcal{L}_{\mathrm{R}} \mathrm{d}^{4} x=0
$$

with the Lagrangian density

$$
\mathcal{L}_{\mathrm{R}}=\sum_{k=1}^{N} \bar{\psi}_{k}(x)\left(\mathrm{i} \gamma^{\mu} \partial_{\mu}-m_{k}-q_{k} \chi_{0}(x)\right) \psi_{k}(x)+\frac{1}{2} \int \mathrm{d}^{4} x^{\prime} \rho\left(x^{\prime}\right) D\left(x-x^{\prime}\right) \rho(x),
$$

provided that the Green function is symmetric:

$$
D\left(x-x^{\prime}\right)=D\left(x^{\prime}-x\right) .
$$

We shall consider the QFT based on the Lagrangian density (10). Note that there are two interaction terms in this formulation of the Yukawa theory: a "local" interaction containing $\chi_{0}$, and a "nonlocal" one given by the last term of eq. (10). The latter contains the boson propagator explicitly. Thus, in the conventional covariant perturbation theory it leads to Feynman diagrams without external (physical) boson lines. The "local" term is of the conventional form and can be used to generate Feynman diagrams that cannot be generated by the "nonlocal" interaction term, particularly diagrams with external boson lines. Of course, care must be taken not to double the physical effects count.

We shall not pursue the covariant perturbation theory. Rather, we shall consider an approach that leads to exact few-fermion eigenstates in the Hamiltonian formalism of the QFT (a brief review of the Hamiltonian method in treating fewbody bound and quasi-bound states is given in [3]). In the Hamiltonian method one seeks solutions of the QFT equation $P^{\mu} P_{\mu}|\Psi\rangle=M^{2}|\Psi\rangle$ where $P^{\mu}=(H, \mathbf{P})$ is a QFTheoretic 4-momentum operator, and $M$ is the invariant mass of the system under study. However, this equation is complicated because of the appearance of quadratic Hamiltonian and momentum operators, so it is customary to do the calculation in the rest frame of the system, $\mathbf{P}|\Psi\rangle=0$, whereupon solutions of the equation $H|\Psi\rangle=M|\Psi\rangle$ are sought, as they are evidently solutions of the covariant quadratic equation in this frame.

\section{Hamiltonian in the canonical equal-time formulation and few-fermion states}

For the sake of simplicity we shall consider the case of only two different fermion fields denoted by $\psi_{1}=\psi$ and $\psi_{2}=\phi$, with masses and "charges" $m_{1}=m, q_{1}=$ $q$ and $m_{2}=M, q_{2}=Q$, respectively. We consider this theory in the canonical equal-time formalism. Equal-time quantization corresponds to the imposition of anticommutation rules for fermion fields, namely:

$$
\left\{\psi_{\alpha}(\mathbf{x}, t), \psi_{\beta}^{\dagger}(\mathbf{y}, t)\right\}=\left\{\phi_{\alpha}(\mathbf{x}, t), \phi_{\beta}^{\dagger}(\mathbf{y}, t)\right\}=\delta_{\alpha \beta} \delta^{3}(\mathbf{x}-\mathbf{y})
$$


and all the others vanish. In addition, there are usual commutation rules for the $\chi_{0}$ field, and commutation of $\chi_{0}$ field operators with $\psi$ and $\phi$ field operators.

We choose to work in the Schrödinger picture where we can take $t=0$ in the expressions for field operators, that is $\psi(x)=\psi(\mathbf{x}, t=0), \rho(x)=\rho(\mathbf{x}, t=0)$, etc. Therefore, for the symmetric ("principal value") Green function of equation (7) it follows that

$$
\int_{-\infty}^{\infty} \mathrm{d} t^{\prime} D\left(x-x^{\prime}\right)=\frac{\mathrm{e}^{-\mu\left|\mathbf{x}-x^{\prime}\right|}}{4 \pi\left|\mathbf{x}-x^{\prime}\right|}
$$

In that case, the "nonlocal" term of the Hamiltonian density corresponding to the Lagrangian (10) takes on the form:

$$
\mathcal{H}_{\mathrm{I}}(\mathbf{x})=\frac{1}{8 \pi} \int \mathrm{d}^{3} x^{\prime} \rho\left(\mathbf{x}^{\prime}\right) \rho(\mathbf{x}) \frac{\mathrm{e}^{-\mu\left|\mathbf{x}-x^{\prime}\right|}}{\left|\mathbf{x}-x^{\prime}\right|} .
$$

We shall suppress the "local" interaction term that contains $\chi_{0}$ in what follows. Such a truncated model is suitable for describing few fermion states without decay or annihilation involving physical bosons. Thus, the (normal-ordered) Hamiltonian for our model, in the Schrödinger picture with $t=0$, is given by the expression

$$
H_{\mathrm{R}}=H_{\psi}+H_{\phi}+H_{\mathrm{I}}
$$

where

$$
\begin{aligned}
& H_{\psi}=\int \mathrm{d}^{3} x \psi^{\dagger}(\mathbf{x}, 0)(-\mathrm{i} \vec{\alpha} \cdot \nabla+m \beta) \psi(\mathbf{x}, 0), \\
& H_{\phi}=\int \mathrm{d}^{3} x \phi^{\dagger}(\mathbf{x}, 0)(-\mathrm{i} \vec{\alpha} \cdot \nabla+M \beta) \phi(\mathbf{x}, 0),
\end{aligned}
$$

and $H_{\mathrm{I}}$ is given by

$$
\begin{aligned}
H_{\mathrm{I}} & =-\frac{1}{8 \pi} \int \mathrm{d}^{3} x d^{3} x^{\prime} \frac{\mathrm{e}^{-\mu\left|\mathbf{x}-x^{\prime}\right|}}{\left|\mathbf{x}-x^{\prime}\right|} \\
& \times\left[q^{2} \bar{\psi}\left(\bar{\psi}^{\prime} \psi^{\prime}\right) \psi+Q q \bar{\phi}\left(\bar{\psi}^{\prime} \psi^{\prime}\right) \phi+q Q \bar{\psi}\left(\bar{\phi}^{\prime} \phi^{\prime}\right) \psi+Q^{2} \bar{\phi}\left(\bar{\phi}^{\prime} \phi^{\prime}\right) \phi\right],
\end{aligned}
$$

where $\psi=\psi(\mathbf{x})$ and $\bar{\phi}^{\prime}=\bar{\phi}\left(\mathbf{x}^{\prime}\right)$, etc.

We define the unconventional (or "empty") vacuum state $|\tilde{0}\rangle$ by

$$
\psi_{\alpha}(\mathbf{x}, 0)|\tilde{0}\rangle=\phi_{\alpha}(\mathbf{x}, 0)|\tilde{0}\rangle=0
$$

This unconventional empty vacuum definition (19) means that $\psi(\mathbf{x})$ is interpreted as a (free) Dirac-particle annihilation operator, while $\psi^{\dagger}(\mathbf{x})$ is, correspondingly, a Dirac-particle creation operator. By a "Dirac-particle" we mean one described by the full Dirac spinor, including positive- and negative-frequency components. (Recall that in the conventional approach, i.e. using a Dirac "filled negative-energy sea" vacuum, it is only the negative-frequency component of $\psi$ that is an antiparticle creation operator, and the positive-frequency component of $\psi^{\dagger}$ that is a particle creation operator). 
Note that the normal ordering is achieved by using the anticommutation relations (12), as usual, but it is not identical to the conventional normal ordering because of the unconventional definition of $\psi$ and $\phi$ as annihilation operators and of $\psi^{\dagger}, \phi^{\dagger}$ as creation operators.

We note that the state defined by

$$
|1\rangle=\int \mathrm{d}^{3} x \psi^{\dagger}(\mathbf{x}) F(\mathbf{x})|\tilde{0}\rangle
$$

where $F(\mathbf{x})$ is a $4 \times 1$ c-number coefficient vector, is an eigenstate of $H_{\mathrm{R}}$, provided that $F(\mathbf{x})$ satisfies the equation

$$
(-\mathrm{i} \vec{\alpha} \cdot \nabla+m \beta) F(\mathbf{x})=E F(\mathbf{x}),
$$

which is a usual time-independent one-particle Dirac equation (with positive and negative energy solutions), so that $F(\mathbf{x})$ is a Dirac spinor. Therefore, we refer to 1) as a one-Dirac-fermion state.

Similarly, the two-Dirac-particle state,

$$
|2\rangle=\int \mathrm{d}^{3} x \mathrm{~d}^{3} y F_{\alpha \beta}(\mathbf{x}, \mathbf{y}) \psi_{\alpha}^{\dagger}(\mathbf{x}) \phi_{\beta}^{\dagger}(\mathbf{y})|\tilde{0}\rangle,
$$

(summation on $\alpha, \beta=1,2,3,4$ implied) is an eigenstate of $H_{\mathrm{R}}$, provided that the $4 \times 4$ eigenmatrix $F$ satisfies the equation

$$
h_{m}(\mathbf{x}) F(\mathbf{x}, \mathbf{y})+\left[h_{M}(\mathbf{y}) F^{\mathrm{T}}(\mathbf{x}, \mathbf{y})\right]^{\mathrm{T}}+V(\mathbf{x}-\mathbf{y}) \gamma^{0} F(\mathbf{x}, \mathbf{y}) \gamma_{0}^{\mathrm{T}}=E F(\mathbf{x}, \mathbf{y}),
$$

where $h_{m}(\mathbf{x})=-\mathrm{i} \vec{\alpha} \cdot \nabla_{x}+m \beta, \quad V(r=|\mathbf{r}|)=-\frac{q Q}{4 \pi} \frac{\mathrm{e}^{-\mu r}}{r}$, and the superscript $T$ indicates the transpose of the matrix in question. Equation (23) is a two-fermion Dirac-like or Breit-like equation with positive and negative energy solutions, and is, in this respect, different from those obtained in the conventional approach [4] in which the negative-energy components are effectively projected out. In the rest frame of the two-fermion system (i.e. when $|2\rangle$ is taken to be an eigenstate of the momentum operator for this QFT, with eigenvalue 0), equation (23) reduces to an analogous equation in the single relative co-ordinate $\mathbf{r}=\mathbf{x}-\mathbf{y}$ :

$$
h_{m}(\mathbf{r}) F(\mathbf{r})+\left[h_{M}(-\mathbf{r}) F^{\mathrm{T}}(\mathbf{r})\right]^{\mathrm{T}}+V(\mathbf{r}) \gamma^{0} F(\mathbf{r}) \gamma_{0}=E F(\mathbf{r}) .
$$

It is, therefore, equivalent to 16 ordinary, coupled, first-order differential equations for states of the given $J^{P}$ (not all of which are independent). The reduction of such Breit-like equations to a radial form has been discussed by various authors [5-8] and will not be repeated here. Such equations can at least be solved numerically.

Generalizations to systems of more than two fermions are readily obtained. Thus, the three-fermion state, corresponding to the system like $\left|e^{-} e^{-} \mu^{+}\right\rangle$(with scalar exchange interactions in this case) defined by

$$
|3\rangle=\int \mathrm{d}^{3} x_{1} d^{3} x_{2} d^{3} x_{3} F_{\alpha_{1} \alpha_{2} \alpha_{3}}\left(\mathbf{x}_{1}, \mathbf{x}_{2}, \mathbf{x}_{3}\right) \psi_{\alpha_{1}}^{\dagger}\left(\mathbf{x}_{1}\right) \psi_{\alpha_{2}}^{\dagger}\left(\mathbf{x}_{2}\right) \phi_{\alpha_{3}}^{\dagger}\left(\mathbf{x}_{3}\right)|\tilde{0}\rangle
$$


is an exact eigenstate of $H_{\mathrm{R}}$ with eigenvalue $E_{3}$, provided that the $4^{3}=64$ coefficient functions $F_{\alpha_{1} \alpha_{2} \alpha_{3}}\left(\mathbf{x}_{1}, \mathbf{x}_{2}, \mathbf{x}_{3}\right)$ satisfy the three-body Dirac-like equation

$$
\begin{aligned}
& {\left[h_{m}\left(\mathbf{x}_{1}\right)\right]_{\alpha_{1} \alpha} F_{\alpha \alpha_{2} \alpha_{3}}\left(\mathbf{x}_{1}, \mathbf{x}_{2}, \mathbf{x}_{3}\right)+\left[h_{m}\left(\mathbf{x}_{2}\right)\right]_{\alpha_{2} \alpha} F_{\alpha_{1} \alpha \alpha_{3}}\left(\mathbf{x}_{1}, \mathbf{x}_{2}, \mathbf{x}_{3}\right)} \\
& +\left[h_{M}\left(\mathbf{x}_{3}\right)\right]_{\alpha_{3} \alpha} F_{\alpha_{1} \alpha_{2} \alpha}\left(\mathbf{x}_{1}, \mathbf{x}_{2}, \mathbf{x}_{3}\right) \\
& -\frac{q Q}{4 \pi} \frac{\mathrm{e}^{-\mu\left|\mathbf{x}_{3}-\mathbf{x}_{1}\right|}}{\left|\mathbf{x}_{3}-\mathbf{x}_{1}\right|}\left(\gamma^{0}\right)_{\alpha_{1} \alpha}\left(\gamma_{0}\right)_{\alpha_{3} \beta} F_{\alpha \alpha_{2} \beta}\left(\mathbf{x}_{1}, \mathbf{x}_{2}, \mathbf{x}_{3}\right) \\
& -\frac{q Q}{4 \pi} \frac{\mathrm{e}^{-\mu\left|\mathbf{x}_{3}-\mathbf{x}_{2}\right|}}{\left|\mathbf{x}_{3}-\mathbf{x}_{2}\right|}\left(\gamma^{0}\right)_{\alpha_{2} \alpha}\left(\gamma_{0}\right)_{\alpha_{3} \beta} F_{\alpha_{1} \alpha \beta}\left(\mathbf{x}_{1}, \mathbf{x}_{2}, \mathbf{x}_{3}\right) \\
& -\frac{q^{2}}{4 \pi} \frac{\mathrm{e}^{-\mu\left|\mathbf{x}_{1}-\mathbf{x}_{2}\right|}}{\left|\mathbf{x}_{1}-\mathbf{x}_{2}\right|}\left(\gamma^{0}\right)_{\alpha_{1} \alpha}\left(\gamma_{0}\right)_{\alpha_{2} \beta} F_{\alpha \beta \alpha_{3}}\left(\mathbf{x}_{1}, \mathbf{x}_{2}, \mathbf{x}_{3}\right) \\
& =E_{3} F_{\alpha_{1} \alpha_{2} \alpha_{3}}\left(\mathbf{x}_{1}, \mathbf{x}_{2}, \mathbf{x}_{3}\right),
\end{aligned}
$$

where summation on repeated spinor indices is implied. In the rest frame of the three-body system the equations depend only on two independent vectors, however, the reduction of these equations for states of the given $J^{P}$ is more formidable than in the two-body case. Even then one is left with the full complexity of a relativistic three-body system.

\section{Some explicit solutions for the two-body case}

The $4 \times 4$ matrix equation (24) for the relative motion of the two-fermion system is very similar to that derived previously [8] for a related model, namely, Coulomb QED (QED in the Coulomb gauge, with the "transverse photon" interaction, $-\alpha \cdot \mathbf{A}$, turned off). That model [8] is a "vector coupling" analogue of the present Yukawa model with $\mu=0$. Equation (24) is the same as equation (26) in [8] if $\gamma^{0} \rightarrow 1$ in the present equation (24). Therefore, the reduction of equation (24) is essentially the same as presented in [8], and will not be repeated here. We shall, however, recount some salient points. In the first place equation (24) has the Schrödinger equation as a non-relativistic limit, and the Dirac equation (with a scalar coupling in this case) as a one-body limit if one of $m_{i} \rightarrow \infty$. If we write

$$
F(\mathbf{r})=\left[\begin{array}{ll}
s(\mathbf{r}) & t(\mathbf{r}) \\
u(\mathbf{r}) & v(\mathbf{r})
\end{array}\right],
$$

where $s, t, u, v$ are each $2 \times 2$ matrices, then for $J=0$ states, which are the only ones that we shall discuss here in some detail, they take on the form $s(\mathbf{r})=\frac{1}{r} s(r) \varphi(\hat{\mathbf{r}})$, and similarly for $t, u, v$, where $\varphi(\hat{\mathbf{r}})$ are $2 \times 2$ spherical bi-harmonics defined in [8]. The number of coupled radial equations is generally six for singlets and uncoupled triplet states, and eight for coupled triplet states (of the type $1^{-}::^{3} S_{1}+{ }^{3} D_{1}$ ), though not all of them are independent. It turns out that there are only four radial equations in the $J=0$ case. They are explicitly

$$
\left(m_{1}+m_{2}+V(r)-E\right) s(r)-t^{\prime}(r)-\frac{K}{r} t(r)-u^{\prime}(r)-\frac{K}{r} u(r)=0
$$




$$
\begin{gathered}
\left(m_{1}-m_{2}+\eta V(r)-E\right) t(r)+s^{\prime}(r)-\frac{K}{r} s(r)+v^{\prime}(r)-\frac{K}{r} v(r)=0 \\
\left(-m_{1}+m_{2}+\eta V(r)-E\right) u(r)+s^{\prime}(r)-\frac{K}{r} s(r)+v^{\prime}(r)-\frac{K}{r} v(r)=0 \\
\left(-m_{1}-m_{2}+V(r)-E\right) v(r)-t^{\prime}(r)-\frac{K}{r} t(r)-u^{\prime}(r)-\frac{K}{r} u(r)=0
\end{gathered}
$$

where $s^{\prime}=\frac{\mathrm{d} s}{\mathrm{~d} r}, V(r)=-\frac{q_{1} q_{2}}{4 \pi} \frac{\mathrm{e}^{-\mu r}}{r}, E$ is the eigenenergy (two-particle bound-state mass) to be determined, while $K=1$ for the $0^{-}\left({ }^{1} S_{0}\right)$ states and $K=-1$ for the $0^{+}\left({ }^{3} P_{0}\right)$ states. The parameter $\eta=-1$ for the present Yukawa model for which the coupling is with a scalar mediating field. However, if $\eta=1$, then for $\mu=0$ we recover the equations for the "vector coupling" Coulomb QED model discussed in [8].

Equations (28)-(31) are not independent. Indeed, elementary manipulations of these equations show that

$$
v(r)=\frac{E-m_{1}-m_{2}-V(r)}{E+m_{1}+m_{2}-V(r)} s(r), \quad u(r)=\frac{E-m_{1}+m_{2}-\eta V(r)}{E+m_{1}-m_{2}-\eta V(r)} t(r),
$$

so that the four equations (28)-(31) reduce to only two:

$$
\begin{gathered}
s^{\prime}(r)=\left(\frac{K}{r}+R(r)\right) s(r)+P_{t}(r) t(r), \\
t^{\prime}(r)=-\left(\frac{K}{r}+Q(r)\right) t(r)+P_{s}(r) s(r),
\end{gathered}
$$

where

$$
\begin{gathered}
R(r)=\frac{\left(m_{1}+m_{2}\right) V^{\prime}(r)}{(E-V(r))\left(E-V(r)+m_{1}+m_{2}\right)} \rightarrow \bar{R}=0, \\
Q(r)=\frac{\left(m_{2}-m_{2}\right) \eta V^{\prime}(r)}{(E-\eta V(r))\left(E-\eta V(r)+m_{1}-m_{2}\right)} \rightarrow \bar{Q}=0, \\
P_{t}(r)=\frac{\left(E-V(r)+m_{1}+m_{2}\right)\left(E-m_{1}+m_{2}-\eta V(r)\right)}{2(E-V(r))} \rightarrow \bar{P}_{t}=2 m_{2}, \\
P_{s}(r)=-\frac{\left(E-V(r)-m_{1}-m_{2}\right)\left(E+m_{1}-m_{2}-\eta V(r)\right)}{2(E-\eta V(r))} \\
\rightarrow \bar{P}_{s}=-\frac{m_{1}}{m_{1}+m_{2}}(\bar{\epsilon}-V(r))
\end{gathered}
$$

where $\epsilon=E-m_{1}-m_{2}$ and the quantities with overbars are non-relativistic limits $\left(p / m_{i} \ll 1\right)$.

In general, for arbitrary mass $\mu$ of the mediating field, equations (33) and (34) must be solved numerically. For the case $\mu=0$ (massless quantum exchange) equations (28)-(31), and also (33) and (34), have the well known analytical solutions in the non-relativistic limit. These analytical solutions can be used to obtain 
$O\left(\alpha^{4}\right)$ perturbative corrections to the non-relativistic result $\bar{\epsilon}=-\frac{1}{2} m_{\mathrm{r}} \frac{\alpha^{2}}{n^{2}}$, where $\alpha=q_{1} q_{2} /(4 \pi)$ and $m_{\mathrm{r}}=m_{1} m_{2} /\left(m_{1}+m_{2}\right)$, by using the expression

$$
\epsilon=E-\left(m_{1}+m_{2}\right)=\bar{\epsilon}+\Delta \epsilon
$$

where

$$
\Delta \epsilon=\frac{\langle\bar{\psi}|\Delta H| \bar{\psi}\rangle}{\langle\bar{\psi} \mid \bar{\psi}\rangle}
$$

with $\langle\bar{\psi}|=[\bar{s}(r), \bar{t}(r)], \quad \bar{t}(r)=\frac{1}{2 m_{2}}\left(\bar{s}^{\prime}(r)-\frac{K}{r} \bar{s}(r)\right)$ and

$$
\langle\bar{\psi} \mid \bar{\psi}\rangle=\int_{0}^{\infty}\left[\bar{s}^{2}(r)+\bar{t}^{2}(r)\right] \mathrm{d} r
$$

etc., and also $\left(\mu_{1}=m_{1} /\left(m_{1}+m_{2}\right)\right)$

$$
\Delta H=\frac{1}{\mu_{1}}\left[\begin{array}{cc}
P_{s}+\mu_{1}(\epsilon-V) & -Q \\
-R & \mu_{1}(\epsilon-\bar{\epsilon})+2 m_{2}-P_{t}
\end{array}\right] .
$$

Evaluating (40) to the lowest order in $\alpha$ gives the following results for the lowest $0^{-}$state

$$
\begin{aligned}
& \Delta \epsilon_{\mathrm{S}}\left(n=1{ }^{1} S_{0}\right)=\frac{1}{8} m_{\mathrm{r}} \alpha^{4}\left(3-\frac{m_{\mathrm{r}}}{m_{1}+m_{2}}\right), \\
& \Delta \epsilon_{\mathrm{V}}\left(n=1{ }^{1} S_{0}\right)=\frac{1}{8} m_{\mathrm{r}} \alpha^{4}\left(\frac{7 m_{\mathrm{r}}}{m_{1}+m_{2}}-1\right),
\end{aligned}
$$

where subscript $S$ stands for the "scalar coupling" (the present Yukawa model with $\mu=0$ ), while $V$ stands for the "vector coupling" (that is, the Coulomb QED model treated in [8]). It is of interest to note that these results agree, to this order, with the previously derived expressions that used a conventional approach (with the Dirac "filled negative energy sea" vacuum (see [9] for $\Delta \epsilon_{V}$, and [4] for $\Delta \epsilon_{S}$ ). Note also, that expressions (43) and (44) have correct $O\left(\alpha^{4}\right)$ one-body Dirac limits (one of $m_{i} \rightarrow \infty$ ) which, according to [10], are:

$$
E_{\mathrm{S}}=\frac{m}{\sqrt{1+\alpha^{2}}}=m\left(1-\frac{1}{2} \alpha^{2}+\frac{3}{8} \alpha^{4}+\cdots\right)
$$

for the scalar coupling and

$$
E_{\mathrm{V}}=m \sqrt{1-\alpha^{2}}=m\left(1-\frac{1}{2} \alpha^{2}-\frac{1}{8} \alpha^{4}+\cdots\right)
$$

for the vector coupling.

The analogous results from equations (39)-(42) for the lowest, $0^{+}\left(2^{3} P_{0}\right)$, equal mass $\left(m_{1}=m_{2}=m\right)$ state are

$$
E_{\mathrm{S}}=m\left(2-\frac{1}{16} \alpha^{2}+\frac{49}{3072} \alpha^{4}+\cdots\right)
$$


and

$$
E_{\mathrm{V}}=m\left(2-\frac{1}{16} \alpha^{2}-\frac{23}{3072} \alpha^{4}+\cdots\right) .
$$

Once again, these agree to $O\left(\alpha^{4}\right)$ with the conventionally derived values $[4,9]$.

Equations (33) and (34) were also solved numerically for the lowest $J=0$ states in the equal mass case $\left(m_{1}=m_{2}=m\right.$ ) with $\mu=0$ (the Maple RungeKutta program was used for this purpose). The results for the ground state are given in table 1 . It is seen that $E(\alpha)$ for this two-body system has a behaviour analogous to that obtained in a one-body limit, equation (45). Thus, for the ground state, the mass $E$ of the two-fermion system starts from the perturbative value $E / m=2-\alpha^{2} / 4+11 \alpha^{4} / 64$ at low $\alpha$, and then decreases monotonically to an apparent asymptotic value of unity for large $\alpha$. Note, from equation (45), that this asymptotic value is zero in a one-body limit (when one of $m_{i} \rightarrow \infty$ ). Note also, that there is no critical value of $\alpha$ beyond which $E$ ceases to be real (as it is in the case of a vector coupling). In the latter case (vector coupling) $E(\alpha)$ is real only for $\alpha \leqslant \alpha_{\mathrm{c}}$, where for the ground state $\alpha_{\mathrm{c}}=2$ in the equal-mass case [8] and $\alpha_{\mathrm{c}}$ decreases to unity in a one-body limit (which is the well-known Dirac result, cf. equation (46)).

Table 1. Ground state $n=1,0^{-}\left({ }^{1} S_{0}\right)$ values of $E_{2} / m$ for $m_{1}=m_{2}=m$ and $\mu / m=0$.

\begin{tabular}{|c|l|l|}
\hline$\alpha$ & \multicolumn{1}{|c|}{$\begin{array}{c}\text { Numeric } \\
\text { (equations (33-34)) }\end{array}$} & \multicolumn{1}{|c|}{$\begin{array}{c}\text { Perturbative } \\
\text { (equation (43)) }\end{array}$} \\
\hline 0.01 & 1.9999750017180 & 1.9999750017188 \\
0.05 & 1.999376066 & 1.999376074 \\
0.1 & 1.99751674 & 1.99751718 \\
0.5 & 1.945266 & 1.948242 \\
1.0 & 1.83030 & 1.921875 \\
2.0 & 1.50707 & 3.75 \\
5.0 & 1.29345 & \\
10. & 1.15067 & \\
100. & 1.01506 & \\
\hline
\end{tabular}

The behaviour for the lowest $(n=2)$ excited state, $0^{+}\left(2{ }^{3} P_{0}\right)$ is qualitatively similar. These results are given in table $2 . E / m$ again follows the perturbative expression (47) for small values of $\alpha$ and then decreases monotonically with increasing $\alpha$ towards an apparent asymptotic value of about unity. 
Table 2. Excited state $n=2,0^{+}\left({ }^{3} P_{0}\right)$ values of $E_{2} / m$ for $m_{1}=m_{2}=m$ and $\mu / m=0$.

\begin{tabular}{|c|l|l|}
\hline$\alpha$ & \multicolumn{1}{|c|}{$\begin{array}{c}\text { Numeric } \\
\text { (equations (33-34)) }\end{array}$} & \multicolumn{1}{|c|}{$\begin{array}{c}\text { Perturbative } \\
\text { (equation (47)) }\end{array}$} \\
\hline 0.01 & 1.9999937501595 & 1.9999937501595 \\
0.05 & 1.999843849586 & 1.999843849691 \\
0.1 & 1.999376588 & 1.999376595 \\
0.5 & 1.985281 & 1.985372 \\
1.0 & 1.94911 & 1.953450 \\
2.0 & 1.85949 & 2.005208 \\
5.0 & 1.66150 & \\
10. & 1.50241 & \\
100. & 1.168404 & \\
\hline
\end{tabular}

Equations (33) and (34) also have unphysical bound-state eigenvalues of the form $E=\left|m_{1}-m_{2}\right|+\cdots$. Such solutions are a consequence of the use of the "empty" vacuum which leads to the retention of negative-energy solutions in the formalism. This, however, is the price that has to be paid in order to obtain exact eigenstates. Such "unphysical" solutions will arise in any two-body equation that has the Dirac one-body limit (or the Klein-Gordon one-body limit in the case of spinless particles [11]).

\section{Concluding remarks}

We have shown that exact few-fermion eigenstates of the QFTheoretic Hamiltonian can be obtained for a reformulated Yukawa model. These eigenstates correspond fo few-fermion systems without decay or annihilation into physical quanta of the mediating scalar boson field. An unconventional empty vacuum state (in contrast to the Dirac filled-negative-energy-sea vacuum) is used, which results in few-body equations with positive and negative energy solutions. The resulting two- and three-fermion equations are written out explicitly. The two-body equation has the relative-motion Schrödinger equation as a non-relativistic (weak coupling) limit and the Dirac equation as a one-body limit (in which the mass of one of the constituent fermions becomes infinite). Positive-energy solutions of the two-body equations are obtained for the lowest $J=0$ states in the case of a massless boson exchange for any strength of the coupling. The two-body bound-state mass, $E(\alpha)$ 
is found to decrease monotonically from the non-relativistic Schrödinger result to a constant asymptotic value at a strong coupling (high $\alpha$ ). Analytical results for $E(\alpha)$ are obtained to $O\left(\alpha^{4}\right)$ for the $J=0$ states under study. These perturbative results are found to agree to the order $\alpha^{4}$ with the results obtained previously by the conventional approach which uses a Dirac vacuum.

\section{Acknowledgement}

The financial support of the Natural Sciences and Engineering Research Council of Canada for this work is gratefully acknowledged.

\section{References}

1. J. D. Jackson. Classical Electrodynamics. John Wiley, New York, 1975.

2. A. O. Barut. Electrodynamics and Classical Theory of Fields and Particles. Dover, New York, 1980.

3. J. W. Darewych. Ukr. J. Phys. 41, 41 (1996).

4. A. D. Polozov, J. W. Darewych, and L. Di Leo. J. Phys. G 21, 1167 (1995).

5. R. W. Childers, Phys. Rev. D 26, 2902 (1982).

6. J. Malenfant. Phys. Rev. D 38, 3292 (1988); Phys. Rev. A 43, 1233 (1991).

7. T. C. Scott, J. Schertzer, and R. A. Moore. Phys. Rev. A 45, 4393 (1992).

8. J. W. Darewych and L. Di Leo. J. Phys. A 29, 6817 (1996).

9. J. W. Darewych and M. Horbatsch. J. Phys. B: At. Mol. Opt. 22, 973 (1989); 23, 337 (1990).

10. W. Greiner. Relativistic Quantum Mechanics. Springer, New York, 1990.

11. J. W. Darewych. Can. J. Phys. (1998), in press.

\section{Власні стани системи кількох частинок у моделі Юкави}

Ю.Даревич

Факультет фізики та астрономії, Йоркський університет

Торонто, Онтаріо, Канада

Отримано 13 лютого 1998 р.

Розглядається переформулювання моделі Юкави, у якій ферміони взаємодіють через посередництво (масивного або безмасового) скалярного поля. За допомогою коваріянтних функцій Ґріна поле, що переносить взаємодію, виражено у термінах ферміонних полів. Результуючий гамільтоніян теорії містить член, що описує взаємодію, у який явно входить пропагатор поля - носія взаємодії. Показано, що коли можна знехтувати процесами, що включають випромінювання 
фізичних квантів поля-носія, то з використанням нестандартного означення вакуумного стану можна отримати у рамках канонічного одночасового формалізму точні власні стани результуючого гамільтоніяну для декількох ферміонів. Ці власні стани приводять до двота тричастинкових рівнянь Діракового типу із скалярними взаємодіями. Числовим розв'язуванням рівняння на власні значення отримано двоферміонні зв'язані стани для $J=0$. Проведено порівняння із стандартним розглядом цієї моделі.

Ключові слова: системи кількох ферміонів, модель Юкави, спектр мac

PACS: $11.10 . Q r, 11.10 . s t$ 